\title{
Corangiomas en el Instituto Materno Infantil a propósito de un Corangioma Gigante
}

\author{
Alfredo Ernesto Romero Rojas*; Lilia María Sánchez Baracaldo **
}

\section{RESUMEN}

Presentamos los resultados de una serie de 6 corangiomas vistos en el Instituto Materno Infantil de Santafé de Bogotá, entre 1992 y 1999. La incidencia de corangiomas fue de $0.11 \%$. Uno de los casos fue un corangioma gigante asociado a polihidramnios. Otras asociaciones que se encontraron a los corioangioma incluyeron retardo en el crecimiento intrauterino, pre-eclampsia y embarazo múltiple.

PAlabras ClaVE: Corangioma, Polihidramnios, Hidrops fetal, Corangioma Gigante.

\section{ABSTRACT}

We present the results of a series of six chorangiomas found at the Instituto Materno Infantil in Santafé de Bogotá during the period from 1992 to 1999. The incidence of chorangioma was $0.11 \%$. One of these cases was a giant chorangioma associated to polyhydramnios. Another found associations related to chorangiomas included fetal growth retardation, pre-eclampsia and multiple pregnancy.

KEY WORDS: Chorangioma, Polyhydramnios, Hydrops fetalis, Giant Chorangioma

\section{Introducción}

El corangioma es un tumor benigno de la placenta de carácter vascular del cual algunos autores precisan que más que una lesión tumoral es una anormalidad en el desarrollo vascular dentro de la placenta (1). Su incidencia varía de 0.2 a 7.6 por cada 100 placentas (2). La lesión esta compuesta por numerosos vasos pequeños (capilares) originados a partir de tejido embrionario fetal, estos vasos se encuentran embebidos en un estroma escaso de tejido conectivo y casi siempre se localizan cerca de la cara fetal de la placenta. (3). Se han visto asociados a una morbimortalidad importante, llegando a ser la primera de hasta un $18 \%$ (4). También se han visto asociados a polihidramnios, hidrops fetal, retardo del crecimiento intrauterino (RCIU) y muerte fetal (5). En esta oportunidad presentamos los Corangiomas vistos en el Instituto Materno Infantil (IMI) de Santafé de Bogotá, entre Enero de 1992 y Marzo de 1999.

\footnotetext{
* Residente I de Patología. Instituto Nacional de Cancerologia. ** Profesora Asistente. Departamento de Patología. Facultad de Medicina. Universidad Nacional de Colombia. Instituto Materno Infantil.
}

\section{Materiales y Métodos}

Se realizó un estudio retrospectivo revisando los archivos de anatomía patológica del Departamento de Patología del IMI en búsqueda de los especímenes con diagnóstico de corangioma en el período comprendido entre Enero de 1992 y Marzo de 1999. No se incluyeron otras alteraciones del parénquima vascular de la placenta como la corioangiomatosis y la corangiosis. Se revisaron las historias clínicas de dichas pacientes buscando datos como edad materna, número de gestaciones, edad gestacional (EG), peso fetal, enfermedad materna, embarazo múltiple, polihidramnios, muerte fetal, hidrops fetal y presencia de malformaciones fetales.

\section{Casos Clínicos}

Caso 1: Paciente de 17 años, G2 P1 A1 con embarazo de 33 semanas de EG, parto atendido en mayo de 1994, el recién nacido $(\mathrm{RN})$ pesó $1300 \mathrm{~g}$. La placenta pesó $210 \mathrm{~g}$ y midió $15 \times 10 \times 3 \mathrm{~cm}$. No se evidenció ninguna lesión a nivel del plato basal. El Diagnóstico final fue: Placenta del tercer trimestre con un corangioma microscópico.

Caso 2: Paciente de 27 años, G2 P1 A0 con embarazo de 34 semanas de EG e historia de RCIU, parto atendido en julio de 1996 , los RN pesaron $1820 \mathrm{~g}, 1800 \mathrm{~g}$ y 1600 g cada uno. La placenta era triamniótica, pesó 790 g y midió $33 \times 16 \times 2 \mathrm{~cm}$. A los cortes seriados era de color pardo violáceo con algunas áreas blanquecinas. El Diag- 
nostico final fue: Placenta madura del tercer trimestre, bicorial-triamniótica con presencia de un corangioma.

Caso 3: Paciente de 29 años, G3 P2 A1 con embarazo de 39 semanas de EG, pre-eclampsia y RCIU, parto atendido en agosto de 1997, el RN pesó $2310 \mathrm{~g}$. La placenta pesó 470 g y midió 16 x 14 x $1.6 \mathrm{~cm}$. A los cortes seriados se observó hacia uno de los márgenes un área hemorrágica que midió 3 x $4 \mathrm{~cm}$ y ocupó todo el espesor del plato basal. El Diagnóstico final fue: Placenta madura del tercer trimestre circunmarginada el $30 \%$ con villitis crónica no especifica grado histológico II/III, corangioma de $3 \mathrm{~mm}$ y hematoma intervelloso.

Caso 4: Paciente de 43 años, G3 P1 A1 con embarazo de 26 semanas de EG, pre-eclampsia y RCIU, parto atendido en septiembre de 1997 , el RN pesó $600 \mathrm{~g}$. La placenta pesó $150 \mathrm{~g}$ y midió $10 \times 11 \times 1.5 \mathrm{~cm}$. A los cortes seriados se observó un infarto reciente pequeño. El Diagnóstico final fue: Placenta del segundo trimestre con presencia de un infarto reciente, corangioma y abundantes depósitos de fibrina intervellosos. Amnios y cordón umbilical sin alteraciones.

Caso 5: Paciente de 25 años, G2 P1 V1 con embarazo de 34 semanas de EG y pre-clampsia, parto atendido en septiembre de 1997, el RN peso 2260 g. La placenta pesó $510 \mathrm{~g}$ y midió $24 \times 18 \times 4 \mathrm{~cm}$, circunmarginada en un $95 \%$, a los cortes seriados se observó marcada congestión, venia acompañada por una masa multinodular, rojiza con vasos aparentes en su superficie que pesó $237 \mathrm{~g}$ y midió 11 x $8 \mathrm{x}$ $5 \mathrm{~cm}$, al corte la superficie era de aspecto hemorrágico (Foto $\mathrm{N}^{\circ} 1$ ). El Diagnóstico final fue: Placenta madura del tercer trimestre circunmarginada en un $95 \%$ con edema focal de las vellosidades y Corangioma Gigante (CG) subcorial con cambios por torsión y escasas calcificaciones.

Caso 6: Paciente de 22 años, G1 P0 con embarazo de 39 semanas de EG, parto atendido en octubre de 1997, el RN pesó $2880 \mathrm{~g}$. La placenta pesó $470 \mathrm{~g}$ y midió 16 x 12

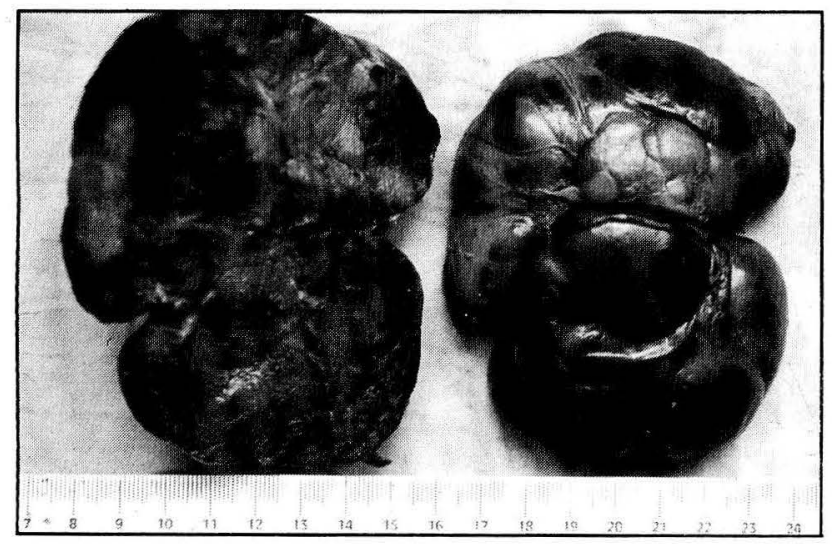

FOTO 1

A LA IZQUIERDA SE EVIDENCIA LA SUPERFICIE EXTERNA DE LA MASA, A LA DERECHA LA SUPERFICIE DE CORTE, EN DONDE LAS ÁREAS BLANQUECINAS CORRESPONDEN A ZONAS DE INFARTO. x $2 \mathrm{~cm}$. A los cortes seriados se observó marcada congestión. El Diagnóstico final fue: Placenta madura del tercer trimestre con corioamnionitis grado histológico II/III, funisitis grado histológico I/III, corioangioma de $1.5 \mathrm{~cm}$ de diámetro mayor.

\section{Resultados}

Entre enero de 1992 y Marzo de 1999 se encontraron 7 reportes de corangiomas, entre 5327 estudios de anatomía patológica que correspondían a placentás del segundo y tercer trimestre enviadas para estudio al Departamento de Patología del IMI. Se descartó uno de los casos ya que no se pudo confirmar el diagnóstico al revisar las láminas. Los 6 casos restantes correspondían al $0.11 \%$, es decir se había visto 1 corangioma (1.1) por cada 1000 placentas. Las edades maternas estaban en un rango comprendido entre los 17 y los 43 años con un promedio de edad de 28.1 años. La paridad oscilaba entre 1 y 3 gestaciones, con un promedio de 2.14 embarazos. Los resultados de la revisión de las historias clínicas se encuentran resumidos en la tabla No.1.

Cinco placentas $(83.3 \%)$ eran del tercer trimestre y sólo una (16.6\%) del segundo trimestre de la gestación. En 5 casos $(83.3 \%)$ el diagnóstico de la lesión se realizó durante el estudio microscópico, sólo fue evidente la lesión al examen macroscópico en un caso (caso No. 5), ya que la masa venia separada del plato placentario. El tamaño de los corangiomas varió entre $1.5 \mathrm{~mm}$ y $11 \mathrm{~cm}$ de diámetro. Uno de estos casos ( $16.6 \%)$, fue reportado como CG $(11 \times 8 \times 5 \mathrm{~cm})$, entendiéndose como gigante aquél cuyo diámetro mayor es superior a los $5 \mathrm{~cm}$. En el CG se encontraron también cambios por torsión y escasas microcalcificaciones. Un caso (16.6\%) fue visto en una placenta de embarazo múltiple, trigemelar bicorial triamniótica. En 2 casos (33.3\%) las placentas fueron extracoriales del tipo circunmarginada, en ningún caso se encontró placenta circunvalata (Tabla No. 2). En el caso No 5 se realizó también estudio de inmunohistoquímica con el anticuerpo para el factor VIII el cual fue positivo confirmando aun más la naturaleza vascular de la lesión. No se encontraron corioangiomas múltiples.

Ninguno de los casos presentados había sido diagnosticado clínicamente o por exámenes paraclínicos durante la gestación, o durante el momento del parto. Aún en el caso No 5 el hallazgo de la masa no planteó esta posibilidad diagnóstica, se pensó inicialmente que trataba de un feto acárdico, sin embargo los hallazgos histológicos descartaron esta posibilidad (Foto $\mathrm{N}^{\circ} 2$ ). En este mismo caso durante la cesárea se documentó además un polihidramnios. Los otros casos no mostraron una asociación clínica especifica.

\section{Discusión}

Los corangiomas fueron descritos por primera vez en 1798 por Clarke, desde entonces se han publicado múltiples trabajos de revisión, que han mostrado incidencias entre 1:9000 hasta 1:50.000 placentas (6). En 1971 Wallenburg informó para esta lesión una incidencia aun mayor; de hasta 1 por 117 placentas (7) y posteriormente, en 1991, Soma encontró la presencia del tumor en el 0.2\% 
TABLA 1

\begin{tabular}{|c|l|l|c|l|}
\hline $\begin{array}{l}\text { CASO } \\
\mathbf{N}^{\circ}\end{array}$ & $\begin{array}{l}\text { EDAD } \\
\text { MATERNA }\end{array}$ & $\begin{array}{l}\text { EDAD } \\
\text { GESTACIONAL }\end{array}$ & PESO FETAL & $\begin{array}{l}\text { ALTERACIONES } \\
\text { ASOCIADAS }\end{array}$ \\
\hline 1 & 17 & 33 semanas & $1300 \mathrm{~g}$ & Ninguna \\
\hline 2 & 27 & 34 semanas & $\begin{array}{r}1820 \mathrm{~g} \\
1800 \mathrm{~g} \\
1600 \mathrm{~g}\end{array}$ & RCIU \\
& & & $2310 \mathrm{~g}$ & RCIU-Pre-eclampsia \\
\hline 3 & 29 & 39 semanas & $600 \mathrm{~g}$ & RCIU-Pre-eclampsia \\
\hline 4 & 43 & 26 semanas & $2260 \mathrm{~g}$ & Pre-eclampsia, Polihidramnios \\
\hline 5 & 25 & 34 semanas & $2880 \mathrm{~g}$ & Ninguna \\
\hline 6 & 22 & 39 semanas & & \\
\hline
\end{tabular}

TABLA 2

\begin{tabular}{|c|c|c|l|}
\hline CASO \# & TAMAÑO & $\begin{array}{l}\text { PLACENTA } \\
\text { EXTRACORIAL }\end{array}$ & $\begin{array}{l}\text { OTROS HALLAZGOS } \\
\text { ANATOMOPATOLOGICOS }\end{array}$ \\
\hline 1 & $1.5 \mathrm{~mm}$ & NO & NO \\
\hline 2 & $1.5 \mathrm{~mm}$ & NO & Placenta trigemelar. \\
\hline 3 & $3 \mathrm{~mm}$ & Circunmarginada & $\begin{array}{l}\text { Villitis crónica inespecifica, Hematoma } \\
\text { intervelloso. }\end{array}$ \\
\hline 4 & $2 \mathrm{~cm}$ & NO & Infarto reciente. \\
\hline 5 & $11 \times 8 \times 5 \mathrm{~cm}$ & Circunmarginada & Edema focal de las vellosidades. \\
\hline 6 & $1.5 \mathrm{~cm}$ & NO & Coriannionitis y Funisitis. \\
\hline
\end{tabular}

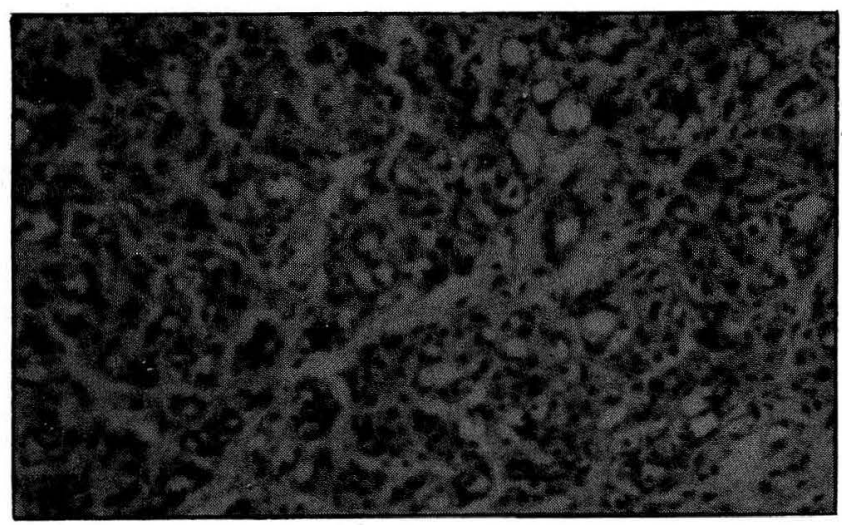

FOTO 2

PROLIFERACIÓN DE VASOS CAPILARES

(H\&E X 40)

de las placentas de mujeres japonesas y hasta en el 7.6\% entre las pobladoras de las grandes alturas en Nepal (2). Sin embargo, debido a la variabilidad en el tamaño de estas lesiones que en su mayoría son milimétricas y se encuentran incluidas dentro del parenquima placentario estas pueden escaparse a la inspección macroscópica, por lo que algunos autores sugieren que la incidencia de estas lesiones puede ser aun mayor (1 caso por cada 100 gestaciones). En esta revisión encontramos que la incidencia de este tumor fue de 1.1 por cada 1000 placentas revisadas en la Unidad de Patología del IMI.

En su serie Shanklin encontró que el tamaño del corangioma capilar oscilaba entre $0.8 \mathrm{~cm}$ y $11.5 \times 9 \times 6 \mathrm{~cm}$, habiendo pesado el tumor en este último caso $258 \mathrm{~g}$ (1). El CG es menos frecuentes y es más fácil de detectar al exámen de la placenta. Este se puede ver como una protuberancia sobre la cara fetal, aun cuando en un pequeño porcentaje puede verse también sobre la cara materna pareciendo estar reemplazando la totalidad o parte de un lóbulo. (3). La superficie externa de estos tumores puede ser lisa brillante o con protuberancias, de color rojo púrpura y se les puede ver además una cápsula (8). En 1985 Arodi informo el caso de un CG que midió $30 \times 20 \times 5 \mathrm{~cm}$, el cual estaba asociado a polihidramnios y abrupcio placentario (9). El tumor más grande que encontramos en esta revisión midió $11 \times 8 \times 5$ $\mathrm{cm}$ y pesó $237 \mathrm{~g}$, esta lesión fue evidente desde el momento del parto, las otras lesiones fueron hallazgos incidentales durante el estudio histológico y tuvieron diámetros que oscilaron entre 0.15 y $2 \mathrm{~cm}$.

En general los corangiomas son redondos y están delimitados por una cápsula delgada, son de color amarillento, café, rojo o blanco y su superficie de corte es lisa 
y firme. Estos tumores están constituidos por vasos pequeños de origen fetal que tienen el tamaño de un capilar, aunque también pueden estar formados por vasos muy dilatados y dar así una apariencia cavernomatosa; estos vasos están separados por escaso tejido conectivo. La lesión siempre esta recubierta por trofoblasto y se localiza en proximidad a la superficie fetal de la placenta (3), aun cuando también se puede encontrar sobre la cara materna. Cuando la masa se ubica junto al cordón umbilical, toma la forma de una neoplasia mixomatosa, debido a la presencia en su proximidad de la gelatina de Wharton. Estos tumores pueden sufrir cambios degenerativos que incluyen necrosis, calcificaciones, cambio mixoide, infartos, hialinización, depósitos de hemosiderina o aún acumulos de grasa (8). En los casos que revisamos, solo el CG presentó necrosis por torsión de su pedículo.

En los estudios de microscopia electrónica se ha visto que estos tumores están constituidos por células endoteliales que se localizan sobre una membrana basal y que pueden estar rodeadas por algunos pericitos. La característica ultraestructural de los capilares es similar a la de los capilares normales. En particular las mitocondrias, el aparato de Golgi y el retículo endoplasmático no son muy sobresalientes. Pueden verse también vesículas picnóticas. Muchas de estas células endoteliales aparecen abombadas y con un aumento aparente de la separación entre los organelos, que sugiere la presencia de líquido intracelular (10).

Los estudios de inmunohistoquímica de los vasos sanguíneos que constituyen los corangiomas muestran positividad para los anticuerpos contra la vimentina y la actina del músculo liso, mientras que la citoqueratina solo es focalmente positiva, a diferencia de lo que ocurre con los vasos sanguíneos en los hemangiomas extraplacentarios donde esta reacción es negativa. Los elementos estromales muestran una tinción similar. Estos resultados indican que los corangiomas se originan de los vasos sanguíneos del plato coriónico y de las vellosidades coriónicas.(11).

La relación entre corangioma y polihidramnios ha sido bien descrita, en 1924 Siddall's observó esta asociación en un CG, sin encontrarse complicaciones derivadas de este hallazgo (12). En 1954 McInroy y Kelsey informaron un caso de polihidramnios con $3400 \mathrm{ml}$ de líquido amniótico asociado a un tumor placentario que pesó 454 gr y midió $10.5 \mathrm{~cm}$ de diámetro mayor. Ellos sugirieron que el tumor representaba un "espacio muerto" que favorecía su exudación (13). Esta misma asociación la encontramos en el caso No 5 donde el corangioma era gigante. Más recientemente, en 1996 Mochizuki hizo referencia a la relación entre polihidramnios y corangioma diagnósticados prenatalmente por ecografía (14), al igual que Chen en 1997 (15) y Guerman en 1998, quienes informaron la asociación entre polihidramnios y Síndrome de Ballantyne (16).

Además de la asociación del CG a polihidramnios, se ha visto la presencia concomitante de hidrops fetal y muerte fetal (17). En 1929 Klaften, reportó un polihidramnios de $6000 \mathrm{ml}$ asociado a un mortinato de $1700 \mathrm{gr}$, él consideró que el tumor había ocasionado un aumento de la resistencia vascular con transudación secundaria. (5). En 1976 Knoth, encontró una placenta de $1150 \mathrm{~g}$ con múltiples coroangiomas, asociada a un mortinato de 3250 g. (18). En
1989 López y Kristoffersen informaron un embarazo con placenta previa asociada a un tumor placentario, postulando que la placenta previa había sido una posible complicación del tumor (19). En 1993 Hirata, reportó el caso de una masa placentaria de $8.8 \mathrm{~cm}$ de diámetro mayor diagnosticada por Doppler cuyo feto desarrolló posteriormente hidrops (13). En 1996 D'Ercole reportó en un embarazo de 19 semanas de EG un corangioma localizado en el zona de inserción del cordón umbilical, el cual fue diagnosticado por ecografía y en el seguimiento el feto también desarrollo hidrops (21), en ese mismo año Reshhetnikova reportó la asociación de corangioma con placenta extracorial circunvalata además de hematocrito fetal de 17\%, el cual mejoró luego de la transfusión intrauterina (22). En la presente revisión no encontramos placentas circunvalatas, sin embargo 2 de nuestros casos ocurrieron en placentas circunmarginadas.

Se ha visto cardiomegalia en los fetos con hidrops, en estos casos se considera que el origen de este problema es una falla cardíaca. Otro hallazgo descrito, en estos fetos ha sido la trombocitopenia, asociada a coagulación intravascular diseminada, esta asociación fue descrita en 1971 por Froehlich, quien además vio que estos tumores se veían con mayor frecuencia en mujeres blancas que en afroamericanas, al igual que en embarazos gemelares o con fetos malformados. (23). Solo en uno de nuestros casos la lesión se encontró en una placenta de embarazo múltiple. En 1989 Limaye y Tchabo observaron la presentación de trombocitopenia materna en una gestación que se asoció a un corangioma de $5 \mathrm{~cm}$ de diámetro y postularon como origen de ésta, la necrosis del angioma (24). En 1992 Drut, decribió la presencia de hemangioendoteliomas y múltiples corangiomas en fetos con síndrome de BeckwithWidemann (25).

Se han visto otras entidades asociadas al corangioma. En 1965 Heggtveit, observó la presencia comcomitante de pre-eclampsia (26); en 1969 Phillippe encontró en 7 casos de corangioma que los fetos eran pequeños para la EG (27). Mientras que en 1986 Stiller y Skafish encontraron hemorragia fetomaterna en una placenta que tenía 8 corangiomas (28), en este caso tal vez el aumento marcado de la circulación fetal había producido los corangiomas, que a su vez llevaron a un RCIU. La presencia de placenta previa y amenaza de parto pretérmino también ha sido asociada a corangioma. En 1968 Asadourian y Taylor observaron asociación a abrupcio placentario (29). Finalmente, también se ha visto en estas pacientes elevación de la hormona gonadotrofina coriónica como la descrita en 1986 por Sulman y Sulman, (30), y en 1998 por Bashiri, quien reportó la presencia de un corangioma en el segundo trimestre del embarazo asociado a la elevación exagerada de los niveles séricos de la hormona gonadotrofina coriónica.(17). En nuestros casos y en particular en el caso No 5, donde el corangioma era gigante, se encontró concomitante al polihidramnios una pre-eclampsia. $\mathrm{Ob}$ servamos asociaciones múltiples en los casos No 3 y 4 donde ocurrieron a la vez pre-eclampsia y RCIU.

\section{Conclusiones}

Los corangiomas son tumores placentarios poco frecuentes cuya incidencia no es posible establecer dadas sus 
características y la falta de un examen macroscópico estandarizado para todas las placentas. Aun cuando la mayoría de estas lesiones son un hallazgo incidental sin implicaciones clínicas directas sobre la vida de la madre o el feto, en algunos casos existen asociaciones clínicas especificas, más aun cuando el tumor llega a ser un CG, es en este último caso, en el que el tumor puede llegar a explicar una muerte fetal intrauterina, un hidrops fetal o un polihidramnios. Finalmente, recordamos que estos tumores también se pueden diagnosticar en el control prenatal mediante estudios ecográficos.

\section{BIBLIOGRAFIA}

1. Shanklin, D.R., Chorangiomas and others tumors. En: Lewis, S.H. and Perrin, E.: Pathology of thae placenta. 2nd Edition. Churchill Livingstone. Mew York, 1999.

2. Soma, H., Satoh, M., Higashi, S., et al: Ultraestructure of choriocarcinoma. J. Clin. Electron Microsc. 24:5-6, 1991.

3. Marchetti, A.A.: A consideration of certain types of benign tumors of the placenta. Surg. Gynecol. Obstet. 68:733-743, 1939.

4. Fournet, P., Eliot, S., et al: Chorangioma of the placenta. Echographic, rediologic and anatomo-pathologic correlations. J. Gynecol Biol Reprod (Paris), 18(7): 913-8, 1989.

5. Klaften, E: Chorionhemangioma placentae. Z. Geburtshilfe Gynakol. 95:426-437, 1929.

6. Fox, H.: Vascular tumors af the plecenta. Obstet. Gynecol. Surv. 22:697-711, 1967.

7. Wallenburg, H.C.S.: Chorioangioma of the placenta: thirteen new cases and a review of the literature from 1939-1970 with especial reference to the clinical complications. Obstet. Gynecol. Surv. 26:411-425, 1971.

8. Bernischke, K., and Kaufmann, P.: Pathology of the Human Placenta. 3rd. Edition. Springer-Verlag. New York, 1995.

9. Arodi. J., Auslander, R., Atad, J., and Abramovici, H.: Case Report: giant chorioangioma of the placenta. Acta Obstet. Gynecol. Scand. 64:91-92, 1985.

10. Kim C.K., Bernirschke, K., Connolly, K.S.: Chorangioma of the placenta. Chromosomal and electron microscopic studies. Obstet Gynecol. 37(3):372-6, 1971.

11. Lifschitz-Mercer, B., Ogel, M., Kushnir, I., et al.: Chorangioma. A cytoskeletal profile. Int J Gynecol Pathol, 8(4):349-356, 1989.

12. Siddall, R.S.: Chorioangiofibroma (chorioangioama). Am. J. Obstet. Gynecol. 8:430-456, 554-568, 1924.

13. McInnroy, R.A., and Kelsey, H.A.: Chorio-angioma (haemangioma of placenta) associated with acute hydramnios. J. Pathol. Bacteriol. 68:519-523, 1954.

14. Mochizuki, T., Nishiguchi, T., Ito, I., et al.: Ante-natal diagnosis of chorangioma of the placenta: MR features. J Comput Assist Tomogr. 20(3):413-6, May-Jun, 1996.

15. Chen C.P., Cherm, S.R., Wang, T.Y., et al.: Pregnacy with concomitant chorangioma and placental vascular malformation with mesenchymal hiperplasia. Hum Reprod, 12(11):2553-6, 1997.

16. Gherman, R.B., Incerpi, M.H., Wing, D.A., et al.: Ballantyne syndrome: is placental ischemia the etiology?. J Mater Fetal Med. Sep-Oct; 7(5):227-9, 1998.
17. Bashiri, A., Maymon, E., Wiznitzner, A., et al.: Chorangioma of the placenta in asociation with early severe polyhydramnios and elevated maternal serum HCG: a case report. Eur J Obstet Gynecol Reprod Biol. 79(1):103-5, 1998.

18. Knoth, M., Rygaard, J., and Hesseldahl, H.:Chorangioma with hydramnios and intrauterine fetal death. Acta Obstet, Gynecol. Scand. 55:279-281, 1976.

19. Lopez, H.B.B., and Kristoffersen, S.E.: Chorioangioma of the placenta. Gynecol. Obstet. Invest. 28:108-110, 1989.

20. Hirata, G.I., Masaki, D.I., O'Toole, M., Medearis, A.L. and Platt, L.D.: Color flow mapping and Doppler velocymetry in the diagnosis and managemente of a placental chorioangioma associated with noninmune fetal hydrops. Obstet. Gynecol. $81: 850-852,1993$.

21. D' Ercole, C., Cravello, L., Boubli, L., et al.: Large chorangioma associated with hydrops fetales: prenatal diagnosis and management. Fetal Diagn Ther. 11(5):357-360, Sept-Oct, 1996

22. Reshetnikova, O.S., Burton, G.J., Milovanov, A.P., et al.: Incrased incidence of placental chorangioma in high-al-titude pregnacies: hypobaric hipoxia as a possible etiologic factor. Am J Obstet Gynecol. 174(2):557-61, 1996.

23. Froehlich, L.A., and Housler, M.: Neonatal thrombo-cytopenia and chorangioma. J.Pediatr.78:516-519, 1971.

24. Limaye, N.S., and Tchabo, J.G.:Asymptomatic thrombocytopenia associated with chorioangioma of placenta. Am. J. Obstet. Gynecol. 161:76-77, 1989.

25. Drut, R., Drut, R.M., and Toulouse, J.C.: Hepatic hemangioendotheliomas, placental chorangiomas, and dysmorfic kidneys in Becwith-Wiedemann syndrome. Pediatr. Pathol. 12:197-203, 1992.

26. Heggtveit, H.A. de Carvalho R., and Nuyens, A.J.: Chorioangioma and toxemia of pregnacy. Am. J. Obstet. Gynecol. 91:291-292, 1965.

27. Philippe, E., Muller, G., Dehalleux, J.M., et al.: Le chorangiome et ses complications fetomaternelles. Rev. Fr. Gynecol. 64:335$341,1969$.

28. Stiller, A.G. and Skafish, P.R.: Placental chorangioma: a rare case of fetomaternal transfusion with maternal hemolysis and fetal distress. Obstet. Gynecol. 67:296-292, 1986.

29. Asadourian, L.A., and Taylor, H.B.: Clinical significance of placental hemangiomas. Obstet. Gynecol. 31:551-555, 1968.

30. Sulman, F.G, and Sulman, E.: Increased gonadotrophin production in a case of detachment of placenta due to placental haemangioma. J. Obstet, Gynecol. Br. Emp. 56:1033-1034, 1949. 